\title{
Thermal Infrared Spectroscopy of Saturn and Titan from Cassini
}

Donald E. Jennings, J, C. Brasunas', R. C. Carlson ${ }^{2}$, F. M. Flasar ${ }^{1}$, V. G. Kunde ${ }^{3}$, A. A. Mamoutkine ${ }^{4}$, C. A. Nixon ${ }^{3}$, J. C. Pearl', P. N. Romani', A. A. Simon-Miller', G. L. Bjoraker', CIRS Tean

NASA/Goddard Space Flight Center, Code 693, Greenbelt, MD 20771, USA

${ }^{2}$ The Catholic University of Amerta, Washington, DC 20064 USA.

${ }^{3}$ University of Maryland, College Park, MD 20742 USA.

${ }^{4}$ Adnet Systems, Inc., Rockville, MD 20852 USA.

\begin{abstract}
Ahstract: The Cassini spacecraft completed its nominal mission at Satum in 2008 and began its extended mission. Cassini carries the Composite Infrared Spectrometer (CIRS), a. Fourier transform spectrometer that measures the composition, thermal stracture and dynamics of the atmospheres of Saturn and Titan, and also the temperatures of other moons and the rings.
\end{abstract}

\section{Introduction}

The Cassini spacecrat began orbiting Saturn in July 2004. The puimary mission lasted until 2008 and an extended mission is now underway. Onboard Cassini is the Composite Intrared Spectrometer (CIRS), a Fourier transfom spectrometer designed to map composition, temperature and dynamics in the atmospheres of Saturn and Titan (Flasar et al, 2004). CIRS infrared mapping is performed at high spectral and spatial resolutions and covers two orders of magnitude in wavelength. On Saturn and Titan global mapping tracks changes as the season progresses toward northern spring. On Titan, CIRS probes altitudes from the stratosphere to the suface. CIRS also maps temperature and composicion of Saturn's ring material and of the moon surfaces. The early portion of the Cassin mission was spent close to the equatorial plane, but recently the orbit has been tilted to allow a closer look near the poles. On Titan nitrile chemistry occurs in the north and on Saturn temperature extremes cause circumpolar thermal structures and a range of chemical abundances.

The completion of Cassini's nominal mission is an occasion to review the accomplishments of CIRS, to assess the inprovements in our understanding of the Saturnian system that have resulted from Cassin, and to anticipate the advancements yet to come in the extended mission.

\section{Description of Instrument and Operations}

CIRS consists of two interferometers that share a telescope and scanning mechanism (Kunde, et al. 1996 ; Flasar ef al. 2004). The far-infrared $\left(10-600 \mathrm{~cm}^{-1}\right)$ is covered with a polarizing interferometer and the midinfrared $\left(600 \mathrm{~m} 1400 \mathrm{~cm}^{-1}\right)$ is covered with a conventional interferometer. The far-irffared uses wire-grid polarizers mounted on mylar and the mid-infrared uses a $\mathrm{KBr}$ beamspliter. Apodized spectral resolution is selected in the range 0.5 to $15 \mathrm{~cm}^{-1}$. In the far-infrared the focal piane uses two thermocouple detectors sensing the sane 3.5-mrad field of view. In the mid-infrared wo 10 -element HgCdTe detector arrays fone photoconductive and the other photovoltaic) observe two adjacent fields of view, each $10 \times 273 \mathrm{mrad}^{2}$. Cube corner retroreflectors are used in the mid-inftared and roof mirrors are used the in the far-inftared. A diode laser reference interferometer is separated from the infrared interferometers, but has its beam in the center of the moving cube corner retroreflector. The instrument housing temperature is controlled at $170 \mathrm{~K}$, and the mid-infrared detectors are cooled to below $80 \mathrm{~K}$. The instrument temperatures are maintained with passive space-viewing radiators. 
Each observation script is designed, tested and uploaded to Cassini well in advance of the actual CIRS observations. The operation mode for each observation is selected from among several with different detector spatial configurations, resolutions, and integration times, and is chosen to optimize the desired science product. Deep space reference spectra for calibration are recorded both within the observation sequence and during non-CIRS operations near the time of the observation. Instrument and spacesraft operational parameters can be chosen to minimize electrical and mechanical interferences. After each observation sequence the recorded interferograms are transmitted to Earth and processed. Calibrated spectra are placed in database and are made avalable the science community.

\section{Results from Saturn and Titan}

CIRS has produced a wide range of new scientific results from observations of Titan, Saturn, its moons and rings (Flasar et al. $2005 a$, b). Atmospheric mapping both at the limb and in nadir show that the nitriles $\mathrm{HCN}, \mathrm{HC}_{3} \mathrm{~N}$ and $\mathrm{C}_{2} \mathrm{~N}_{2}$ are concentrated in the northem region of Titan, particularly above about $40^{\circ}$ North tatitude. Complex hydrocarbons also tend to increase in abandance at nothem latitudes on Titan (Coustenis et al. 2007 ). In a window at $530 \mathrm{~cm}^{-1}$ CIRS penetrates the thick atmospheric haze on Thtan to measure the surface themal emission. In Saturn, hydrocabon abundances increase dramatically at the south pole, apparently due to southward transport during southem summer (Hesman et al. 2008). In the riles, varymg densities and particle sizes can be deduced from temperature distributions (Leyrat ef al. 2008). Thermal maps of Enceladus identify warn fissures at the sites of the southern plumes that are seen in visible images (Spencer etal. 2006).

Figure 1 shows a composite spectrum of Saturn at $0.5 \mathrm{~cm}^{-6}$ resolution from all three focal planes, covering the entire spectral range of CIRS. Saturn spectra are rich in moleculatemission and absorption lines. Among the spectral features seen are absorption from pressure-induced $\mathrm{H}_{2}$, absorption from rotational and vibrational $\mathrm{PH}_{3}$, and emission and absotption from $\mathrm{CH}_{4}, \mathrm{C}_{2} \mathrm{H}_{2}$ and $\mathrm{C}_{2} \mathrm{H}_{6}$. Other features in Saturn spectra but not seen in the figure are absorption from rotational $\mathrm{HD}$ and $\mathrm{NH}_{3}$, and emission from the minor hydrocarbons $\mathrm{C}_{3} \mathrm{H}_{4}$ and $\mathrm{C}_{3} \mathrm{H}_{8}$ and $\mathrm{C}_{4} \mathrm{H}_{2}$. Satum is presently moving seasonally toward northern Vernal Equinox and should begin to exhibit changes in the meridional distribution of minor constituents, The changes in continum tenperature and molecular emssions will be tracked during the extenced Cassini mission.

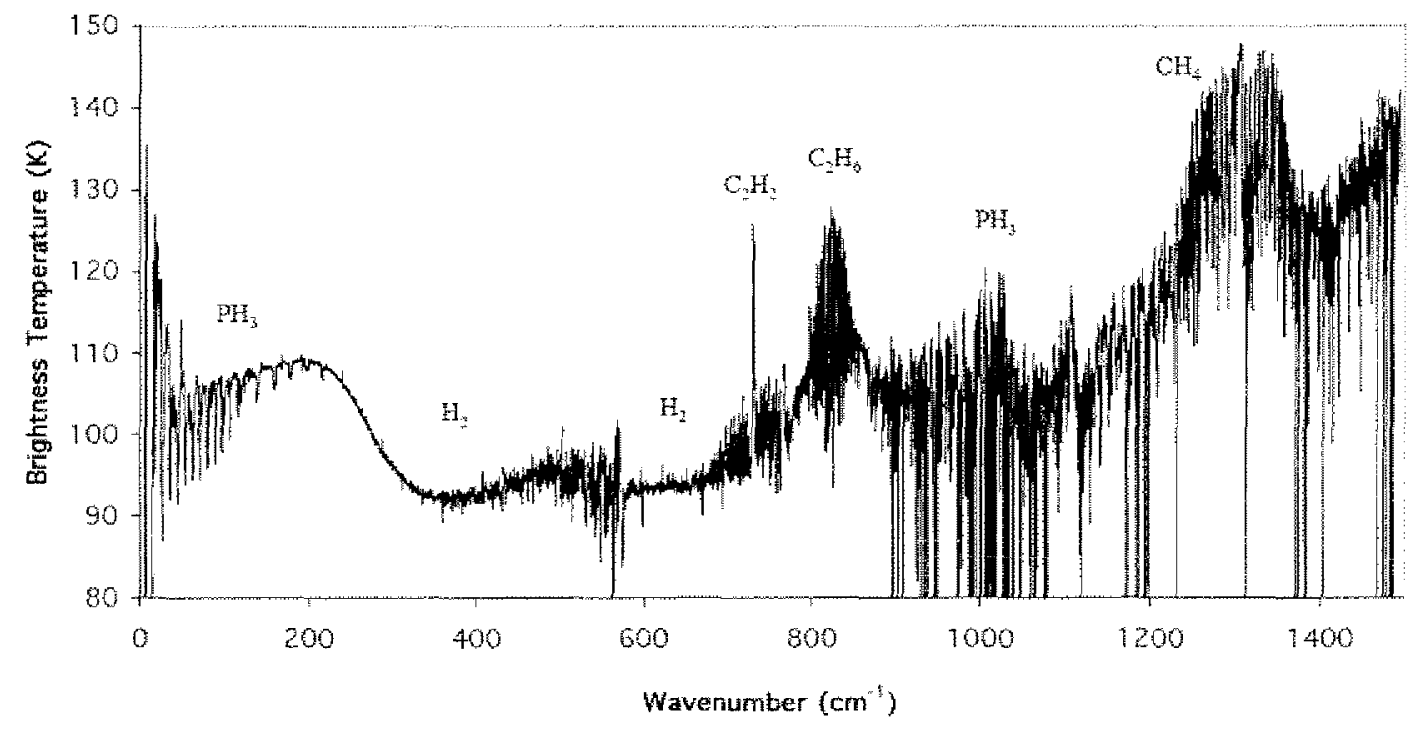

Figure 1. CIRS spectrum of Saturn displayed in brightness temperature. This is a composite spectrum recorded with all three spectral chanels of CIRS and covers the entire spectral range of the instrument. Molecular species in Satum's amosphere are identified by their enission and absorption spectra. The spectral resolution is $0.5 \mathrm{~cm}^{-1}$. The large down ward excursions in the data are due to noise. 
Titan spectra from CIRS show the presence of many hydrocarbons and nitriles. The principal component of Titan's atmosphere is $\mathrm{N}_{2}$, whose pressure-induced spectrum can be seen in CIRS spectra at $60 \mathrm{~cm}^{1}$. A complete list of atmospheric molecules also includes $\mathrm{H}_{2}, \mathrm{CO}, \mathrm{CO}_{2}, \mathrm{CH}_{4}, \mathrm{HCN}, \mathrm{HC}_{3} \mathrm{~N}, \mathrm{CH}_{3} \mathrm{D}, \mathrm{C}_{2} \mathrm{H}_{2}, \mathrm{C}_{2} \mathrm{H}_{4}$, $\mathrm{C}_{2} \mathrm{H}_{6}, \mathrm{C}_{3} \mathrm{H}_{4}, \mathrm{C}_{3} \mathrm{H}_{8}, \mathrm{C}_{4} \mathrm{H}_{2}$, and $\mathrm{H}_{2} \mathrm{O}$. This variety demonstrates the complex photochenistry that takes place in Titan's atmosphere. Sotopic variants of several of these molecules have been detected by CIRS and the measured isotopic enrichments contribute to an understanding of the history of I'itan's atmosphere.

CIRS will continue to collect spectra in the thermal infrared throughout the extended Cassini inission. Combining fesults from CIRS with those from other instruments on Cassini as well as from other spacecraft and ground-based observations is yielding a detailed, ever improving picture of Saturn, Titan and the rest of the Saturnian system.

\section{References}

F. M. Flasar, ef al., "Exploring the Saturn System in the Thermal Infrared: The Composite Infrared Spectroneter," Space Sejence Reviews, 115,169 (2004).

V. Kunde, et al. "Cassin Infrared Fourier Spectroscopic Investigation," in Cassini/Huygens: A Mission to the Saturnian Systems, Proc. SPIE 2803, $162-177$ (1996).

F. M. Flasar et.al., "Temperatures, winds, and composition in the Satum system," Science, 307, 1247 (2005a).

F. M. Flasar et. al, "Titan's Atmospheric Temperatures, Winds, and Composition," Nature, 308, 975 , (2005b).

A. Coustenis, et al., "The composition of Titan's stratosphere from Cassini/CIRS mid-infrared spectra," Icarus, $189,35(2007)$.

B. E. Hesman et al. "Satum's latitudinat $\mathrm{C}_{2} \mathrm{H}_{2}$ and $\mathrm{C}_{2} \mathrm{H}_{6}$ abundance profiles from Cassini/CIRS and groundbased observations," lcarus, submitted (2008).

C. Leyrat ef al., "Infrared observations of Saturn"s rings by Cassini CIRS: Phase angle and local time dependence," Planetary and Space Science, 56, 117 (2008).

J. R. Spencer et al., "Cassini Encounters Enceladus: Background and the Discovery of a South Polar Hot Spot." Science, 311, 1401 (2006). 